\section{Patent ductus arteriosus and pulmonary artery aneurism in a child affected by Loeys-Dietz syndrome}

\author{
Marcello Marcì \\ Cardiology Department, Villa Sofia- \\ Cervello Hospital, Palermo, Italy
}

\begin{abstract}
We report the case of a 3 -year old girl affected by Loeys-Dietz syndrome, a rare association of aneurysms and tortuosity of arteries associated to hypertelorism and cleft palate that was firstly described by Loeys and Dietz in 2005 . The patient developed aneurysms of aortic root and of pulmonary artery, besides a patent ductus arteriosus was evidenced. We also reviewed the current literature concerning the aetiology and management of this syndrome.
\end{abstract}

\section{Introduction}

The rare association of aneurysm and tortuosity of arteries, hypertelorism and cleft palate was reported by Loeys-Dietz in 2005. ${ }^{1,2}$ Since then only few cases have been described. We report the case of a 3-year old girl affected by Loeys-Dietz syndrome (LDS), who developed aneurysms of aortic root and of pulmonary artery, moreover she underwent percutaneous closure of patent ductus arteriosus (PDA).

\section{Case Report}

The patient was the second child of nonconsanguineous and healthy parents, her brother was healthy, too. She was born at term by caesarean section, after an uncomplicated pregnancy. During the first month of life she was referred to our institution for cardiac evaluation for a continuous murmur associated to posterior cleft palate, hypertelorism, and prominent forehead, talipes equinovarus feet were also noticed. Karyotype was $46 \mathrm{XX}$.

The echocardiogram showed a PDA and mild dilatation of the pulmonary artery and of the aortic root, dimensions of cardiac chambers were normal. After discharge the patient was followed every six months. At 1 year of age a progressive worsening of clinical manifestations and gradual progression of aortic aneurysm were evidenced. She was admitted again for a reappraisal of cardiac disease. The infant showed unsatisfying growth (weight was in $3^{\text {rd }}$ percentile, height was at $<10^{\text {th }}$ percentile). Motor and developmental delay became evident. Eye examination revealed only strabismus ruling out ectopia lentis.

Echocardiography showed significant progression in pulmonary artery dilatation (25 $\mathrm{mm}$ ), and aortic root aneurysm (26 mm, Z score: 5) (Figure 1). Furthermore, the shunt through the ductus caused dilatation of left atrium and ventricle (end-diastolic diameter $=37 \mathrm{~mm}$, end-diastolic volume $=75 \mathrm{~mL}$ ).

On the basis of dysmorphic facial appearance, skeletal abnormalities, associated to cardiovascular anomalies, the LDS type I was suspected. Finally, the diagnosis was confirmed by demonstration of TGFBR2 gene mutation. In order to assess other vascular anomalies magnetic resonance (MR) angiography was performed. The MR imaging showed corkscrew tortuosity of vertebral and carotid arteries (Figure 2). Based on clinical studies that have demonstrated efficacy of an antagonist of angiotensin II losartan in preventing further dilatation of aortic aneurysm in individuals with Marfan syndrome, treatment with losartan at a dose of $1 \mathrm{mg} / \mathrm{kg} /$ day was started.

The patient was referred to a tertiary centre for percutaneous closure of the ductus with a PDA 4-6 Amplatzer device. Up to now the 3year old child is asymptomatic. Echocardiogram shows a normal left ventricle volume, the aortic root and pulmonary artery size are stabilized.

\section{Discussion}

LDS is an autosomal dominant connective tissue disease, characterized by ocular hypertelorism, craniosynostosis, cleft palate, bifid uvula, cutaneous anomalies and hypermobility of articular joints. Cardiovascular malformations are dominated by aortic root aneurysm, in almost all individuals, in about half of the patients there are widespread arterial tortuosity, generalized aneurysms and dissections while in about a third of the patients PDA is also associated. Loeys and colleagues subdivided the syndrome in two categories: type I patients have cranio-facial anomalies whereas type II patients may manifest bifid uvula and cutaneous findings. Usually, the type I has a more aggressive and widespread vasculopathy. The syndrome is due to mutations of TGFRB 1 and 2 genes encoding respectively for receptor 1 and 2 of transforming growth factor- $\beta$ (TGF$\beta) .{ }^{1-4}$ In consequence of inactivation of those receptors the TGF- $\beta$ signalling pathway is paradoxically upregulated. As a matter of fact, an increased TGF- $\beta$ activity has been demonstrated in aortic surgical samples from patients who underwent aortic repair. TGF represents a family of cytokines that regulates many cellu-
Correspondence: Marcello Marcì, Cardiology Department, Villa Sofia-Cervello Hospital, piazza Gen. Cascino 26, 90142 Palermo, Italy.

E-mail: marcellomarci60@gmail.com

Key words: Echocardiography; Loyes-Dietz syndrome; pulmonary aneurysm; aortic aneurysm; losartan.

Received for publication: 14 April 2015.

Revision received: 20 June 2015

Accepted for publication: 20 June 2015.

This work is licensed under a Creative Commons Attribution NonCommercial 3.0 License (CC BYNC 3.0).

CCopyright M. Marcì 2015

Licensee PAGEPress, Italy

Cardiogenetics 2015; 5:5269

doi:10.4081/cardiogenetics.2015.5269

lar functions. TGF- $\beta$ has pleiotropic effect on cells of arterial wall, it influences growth, differentiation, migration and apoptosis. ${ }^{5}$ In addition, TGF- $\beta$ is a potent regulator of extracellular matrix formation ${ }^{3-5}$ it particularly stimulates fibroblasts to produce collagen, contributing to vascular fibrosis. TGF- $\beta$ pathway anomaly is involved also in other inheritable connective disorders, such as Goldenhar and Marfan syndrome. This latter, as it is well known, is caused by deficit of fibrillin-1, that is a potent inhibitor of TGF- $\beta$ pathway. Angiotensin II antagonist losartan has demonstrated to inhibit TGF- $\beta$ pathway, because angiotensin II (as well as endotelin, mechanical stress and high glucose levels) exerts activation of TGF- $\beta .{ }^{4}$ In animal models and in some clinical trials in individuals with Marfan syndrome losartan showed to prevent further dilatation of aortic aneurysm. ${ }^{6,7}$ Given that the TGF- $\beta$ pathway is upgraded both in Marfan and in LDS patients, it has been postulated that treatment with Losartan may be useful in individuals with TGFRB mutations. The LDS phenotype overlaps that of Marfan syndrome (aortic aneurysm, aracnodattily, dural ectasia and joint hyperextension) but it has also distinct characteristics, such as hypertelorism, cleftpalate and bifid uvula. In contrast to subjects with Marfan, patients with TGFRB mutations exhibit arterial tortuosity and aneurysm throughout the body and a more rapid evolution to dissection. Noteworthy, aortic dissection or rupture can usually happen at smaller aortic-root diameters in patients suffering from LDS than in those with Marfan disease. Consequently, LDS has a worse prognosis than other collagen tissue disorders, as demonstrated by the Loeys' group. In their cohort of 90 patients the median survival is 37 years, with a mean age of death of 26 years. ${ }^{2}$ Whereas the 


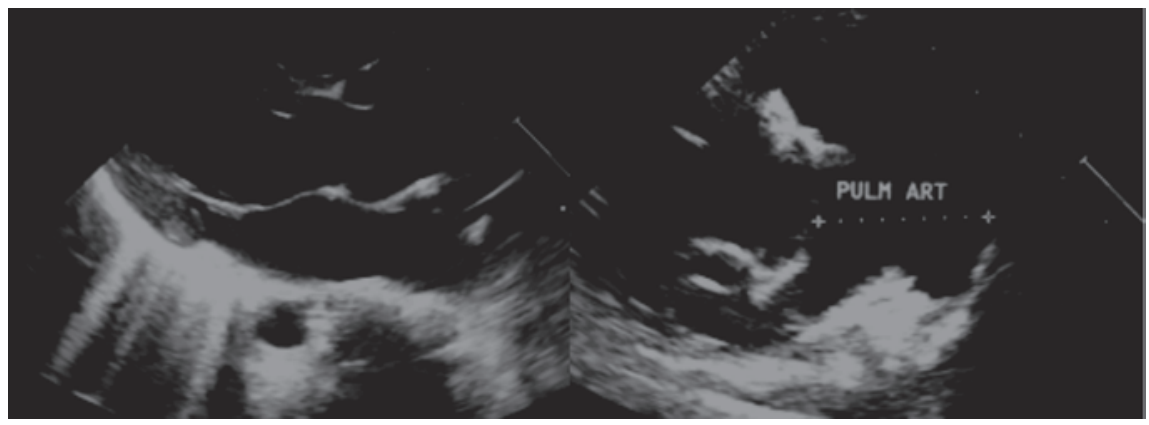

Figure 1. On the left long-axis parasternal view demonstrating aortic aneurysm; on the right parasternal short-axis that shows pulmonary artery aneurysm.

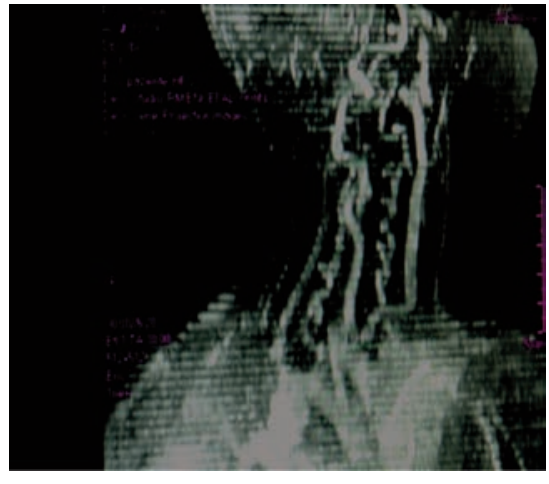

Figure 2. Angio-magnetic resonance demonstrating corkscrew tortuosity of arteries of neck and head.

median survival is about 48 years in patients with Ehlers-Danlos ${ }^{8}$ and 72 years in Marfan syndrome. ${ }^{9}$ However, surgical outcome is better in LDS patients than in subjects affected by Ehlers-Danlos syndrome. ${ }^{10}$ Interestingly, Attias and colleagues demonstrated that survival of individuals with TGFRB mutations is markedly improved by clinical surveillance and specific treatment. ${ }^{11}$ As emphasized by these Authors an early diagnosis of LDS is of crucial importance to improve prognosis, because treatment with losartan may prevent vascular complications. ${ }^{11,12}$ Further clinical trials should be nec-
2. Loyes BL, Schwarze U, Holm T, et al. Aneurysm syndrome caused by mutations in the TGF-beta receptor. N Engl J Med 2006;355:788-98.

3. Annes JP, Munger JS, Rifkin DB. Making sense of latent TGFbeta activation. J Cell Sci 2003;116:217-24.

4. Neptune ER, Frischmeyer PA, Arking DE, et al. Dysregulation of TGFbeta activation contributes to pathogenesis in Marfan syndrome. Nat Genet 2003;33:407-11.

5. Ruiz-Ortega M, Rodríguez-Vita J, SanchezLopez E, et al. TGF- $\beta$ signaling in vascular fibrosis. Cardiovasc Res 2007;74:196-206.

6. Habashi JP, Judge DP, Holm TM, et al. Losartan, an AT1 antagonist, prevents aortic aneurysm in a mouse model of Marfan syndrome. Science 2006;312:117-21.

7. Ramirez F, Dietz HC. Marfan syndrome: from molecular pathogenesis to clinical treatment. Curr Opin Genet Dev 2007;17: 252-8.

8. Pepin M, Schwarze U, Superti-Furga A, Byers PH. Clinical and genetic features of Elhers-Danlos syndrome type IV, the vascular type. N Engl J Med 2000;342:673-80.

9. Silverman DI, Burton KJ, Gray J, et al. Life expectancy in the Marfan syndrome. Am J Cardiol 1995;75:157-60.

10. Williams JA, Loyes BL, Nwakanma LU, et al. Early surgical experience with LoyesDietz: a new syndrome of aggressive thoracic aortic aneurysm disease. Ann Thorac Surg 2007;83:S757-63.

11. Attias D, Stheneur C, Roy C, et al. Comparison of clinical presentations and outcomes between patients with TGFBR2 and FBN1 mutations in Marfan syndrome and related disorders. Circulation 2009;120:2541-9.

1. Loyes BL, Chen J, Neptune ER, et al. A syndrome of altered cardiovascular, craniofacial, neurocognitive and skeletal development caused by mutations in TGFBR 1 or TGFRB2. Nat Genet 2005;37:275-81.
12. Yetman AT, Beroukhim RS, Ivy DD, Manchester D. Importance of the clinical recognition of Loyes-Dietz syndrome in the neonatal period. Pediatrics 2007; 119:e1199-202. 